\title{
SELECCION DE ÁRBOLES PLUS DE CHAÑAR \\ Geoffroea decorticans (Gillies ex Hook. \& Arn) Burkart \\ EN BASE A CARACTERÍSTICAS FENOTÍPICAS DE CRECIMIENTO Y PRODUCCION FRUTAL
}

\author{
Gutiérrez, Braulio ${ }^{4}$; Gacitúa, Sandra ${ }^{5}$ y Villalobos, Enrique ${ }^{2}$
}

\section{RESUMEN}

Se describe el proceso de selección de árboles plus de chañar (Geoffroea decorticans) con características fenotípicas sobresalientes en cuanto a crecimiento y producción de frutos. Se presenta también la evaluación de esos árboles en función de su superioridad fenotípica y ganancias genéticas esperadas.

Las actividades se efectuaron en tres sectores de la región de Atacama y tuvieron por objeto conformar una primera base de individuos selectos, que en el futuro puedan utilizarse como progenitores en un programa de mejoramiento genético orientado a aumentar la productividad frutal de esta especie.

Palabras clave: Geoffroea decorticans, árboles plus, mejoramiento genético

\section{SUMMARY}

This paper describes the selecting process of Chañar (Geoffroea decorticans) plus trees with outstanding phenotypic characteristics in terms of growth and fruit production. It also presents the evaluation of these trees based on their phenotypic superiority and expected genetic gains.

The activities were carried out in three sectors of the Atacama region and aimed to form a first base of selected individuals, which in the future could be used as parents in a genetic improvement program to increase the fruit productivity of this species.

Keywords: Geoffroea decorticans, plus trees, breeding.

\footnotetext{
${ }^{4}$ Investigador, Instituto Forestal Sede Bio Bio. braulio.gutierrez@infor.cl

${ }^{5}$ Investigadores, Instituto Forestal Sede Diaguitas
} 


\section{INTRODUCCIÓN}

El proyecto Antecedentes de Manejo Silvícola-Sanitario para la Producción Sustentable del Fruto del Chañar en la Región de Atacama, desarrollado por el Instituto Forestal (INFOR) con financiamiento del Fondo de Investigación del Bosque Nativo administrado por la Corporación Nacional Forestal (CONAF), contempla dentro de sus objetivos fortalecer el negocio agroalimentario de chañar a través de la selección de material genético de individuos de alta productividad frutal e identificación de áreas potenciales para su repoblamiento. Para tal efecto propone, entre otras acciones, la selección de árboles de chañar que posean características fenotípicas sobresalientes en cuanto acrecimiento y producción de frutos, para poder mantener un stock de árboles selectos que en el futuro puedan utilizarse como progenitores (fuentes de semillas o propágulos) en un programa de mejoramiento genético orientado a aumentar la productividad de la especie.

En este trabajo se da cuenta de las labores de selección de árboles plus, de la evaluación de los mismos y de la estimación de las ganancias genéticas esperadas al utilizarlos como progenitores en un programa de mejoramiento genético.

En el futuro esta información resultará especialmente valiosa para el establecimiento de ensayos de procedencia-progenie a partir de la preselección de los árboles plus con el objetivo de generar huertos semilleros para abastecer de semillas mejoradas a los viveros locales para la producción de plantas que permitan suplementar, enriquecer y masificar con individuos de características fenotípicas selectas de Chañar, en el marco de la Ley 20.283 de fomento a la conservación y manejo del bosque nativo en el país.

En el corto plazo se persigue identificar material genético de mejor calidad (árboles plus) para constituir con ellos la base de futuros programas de mejoramiento que permitan incrementar en forma progresiva el rendimiento productivo de chañar.

\section{OBJETIVOS} de Atacama.

Seleccionar arboles candidatos a plus de chañar con alta productividad frutal en la región

Evaluar los árboles selectos, calcular sus ganacias genéticas esperadas e identificar a los individuos más apropiados para usarlos en programas de mejoramiento genético.

\section{ANTECEDENTES}

El chañar es un arbusto o árbol pequeño que crece en forma natural desde la provincia de Arica, en los valles de Lluta y Azapa, hasta Combarbalá, en la región de Coquimbo, ocupando sitios de clima seco o con muy escasa precipitación, donde se presenta como ejemplares aislados, o formando pequeños grupos puros y densos (Martínez, 1989, cit por Gutiérrez et al., 2013).

La presencia de grandes árboles adultos es cada vez más escasa debido a las cortas indicriminadas para la producción de carbon. Su madera es apropiada para trabajos de carpintería y mueblería, pero debido a la escasez de diametros importantes, solo se usa a nivel local para la confección de muebles rústicos, enseres domésticos, mangos de herramientas y leña. El fruto constituye el principal uso productivo de la especie, ya sea como alimento para ganado caprino, o utilizándolo en la produción de dulces, jarabes o bebidas para consumo humano (Rodriguez et al., 1983, cit por Gutiérrez et al., 2013).

Para ambas utilidades (madera y frutos) existe interés por mejorar su capacidad productiva, especialmente por cuanto el chañar constituye una de las escasas alternativas productivas adaptadas a las adversas condiciones edafoclimáticas imperantes en el norte del país.

En tal sentido, la selección de árboles plus es una de las primeras actividades que 
permite dar inicio a un programa de mejoramiento genético que permita aumentar la productividad de esta especie. La selección de individuos superiores de chañar es el proceso mediante el cual a partir de una población base dada se escogen los mejores árboles, según los rasgos objeto de mejoramiento, para formar o dar origen a la población de mejoramiento y/o de producción. El objetivo inmediato de la selección es suministrar material para establecer pruebas genéticas (procedencias, progenies, clonales, otras), huertos semilleros de plantas o de semillas, huertos semilleros clonales y colectar semilla o material vegetativo.

Estos programas de mejoramiento genético, para ser exitosos deben sustentarse en una base genética amplia, que represente la mayor variabilidad genética posible, y orientarse a obtener la mayor ganancia genética por unidad de tiempo en los caracteres definidos como de interés.

La identificación y selección del árbol de alto rendimiento en cuanto a la variable de interés (madera, frutos, otros), es el inicio y la base fundamental de un programa de mejoramiento genético forestal. La calidad y la rigurosidad de la selección de estos árboles incidirán en la ganancia genética que se alcanzará.

\section{MATERIAL Y MÉTODO}

\section{Identificación del Área de Estudio}

Junto con profesionales de CONAF de la región de Atacama se visitaron varios predios con formaciones boscosas de chañar y fueron seleccionados tres predios ubicados en los sectores de Piedra Colgada, Valle Fértil y San Camilo, todos ubicados a lo largo de la caja del río Copiapó. Estos sectores presentaban árboles aislados con alta productividad frutal y sanitariamente vigorosos.

\section{Selección Árboles Plus}

La selección de los árboles plus se realizó aplicando el método de los árboles de comparación (Ipinza et al., 2000). El método consiste en la comparación del árbol candidato a plus con árboles vecinos, para las características que son objeto del mejoramiento. La comparación se efectuó con respecto a los cinco mejores árboles que existen en la vecindad inmediata que tiene como centro al árbol candidato.

Para la aplicación del método se utiliza un formulario de campo donde se anotan las medidas o puntaje asignados a los árboles de comparación y al árbol candidato. Posteriormente se efectúan los cálculos para obtener el diferencial de selección o el puntaje final que da cuenta de la superioridad del candidato respecto a sus árboles de comparación. Este método tiene la ventaja de que a través de la comparación se elimina el efecto de las diferencias de edad (se comparan árboles coetáneos) y minimiza el efecto de las diferencias de sitio, pues se comparan árboles vecinos que crecen en el mismo micrositio. Como requisito el método requiere que los árboles candidatos y de comparación sean coetáneos, es decir que sean de la misma edad o clase de edad.

Esta metodología permite obtener un diferencial de selección, que se puede interpretar como la ganancia potencial a obtener al seleccionar y utilizar los mejores árboles por fenotipo (Vallejos et al., 2010).

La selección propiamente tal de los árboles candidatos a plus y sus respectivos árboles de comparación fue efectuada por una cuadrilla de tres personas, las que formando una línea perpendicular a la dirección de avance, y separados según densidad del bosquete de chañar por más o menos $50 \mathrm{~m}$, realizó un barrido completo y sistemático de toda la extensión del rodal.

Ante la presencia de un posible árbol sobresaliente candidato a plus, se procedió a revisar el estado sanitario, vigor, copa y producción frutal en todos los planos; analizar su posición social, condición microambiental y competencia; determinar los árboles de comparación; y marcar con cinta al árbol candidato. Si tras este primer análisis en terreno se decide aceptar el árbol como 
candidato a plus, entonces se le asigna un número correlativo por predio, número marcado con pintura de color rojo en dos caras visibles de su fuste, y se le practican dos anillos de pintura para facilitar su detección en el bosque. Simultáneamente se marca con un anillo de pintura y un número correlativo de 1 a 5 los cinco individuos definidos como árboles de comparación. Posteriormente se procedió a referenciar los árboles con GPS y a registrar la información de caracterización de cada árbol de acuerdo a lo requerido en el formulario de selección:

- DAP: Diámetro del fuste a la altura del pecho (1,3 m), de todos los vástagos.

- $\quad$ Altura total: Medida con hipsómetro desde la base al ápice de cada árbol.

- $\quad$ Altura comercial: Medida con hipsómetro desde la base hasta un punto en el fuste en que es posible hacer utilización de la madera.

- Diámetro de Copa: Medido como proyección al suelo en dos direcciones perpendiculares norte-sur y este-oeste.

- Contabilización del número de vástagos y sus diámetros.

- Productividad frutal: Peso seco en kilogramos de todos los frutos de cada árbol.

\section{Evaluación Árboles Plus y Estimación de Ganancias Genéticas}

Con los datos recopilados en terreno se evaluó el porcentaje de superioridad y la ganancia genética estimada, para 76 árboles de chañar seleccionados en rodales naturales, con el objetivo de determinar los más apropiados para incluirlos en programas de mejoramiento genético para biomasa de fuste y producción de frutos. La superioridad fenotípica de todos los árboles seleccionados se calculó utilizando la fórmula siguiente:

\section{$\%$ Superioridad fenotípica $=100 \times(V C-$ VPV $) /$ VPT}

Donde: VC : Valor observado en el árbol candidato

VPV: Valor promedio de los árboles vecinos al candidato

VPT: Valor promedio total (candidato y vecinos)

Cuando el árbol candidato se compara con el promedio de todos los árboles vecinos (incluyendo al candidato) se puede obtener una estimación del diferencial de selección con respecto a todo el rodal, tal como se encuentra en el momento en que se efectúa la selección (Gutiérrez, 2000). El porcentaje de superioridad fenotípica de cada árbol fue calculado para tres variables que se usan para caracterizar atributos de interés de los árboles seleccionados:

Indicador de biomasa del fuste $\left(D^{2} H\right)$ : Se usó como un indicador de la biomasa de fuste de cada árbol $\left(\mathrm{m}^{3}\right)$. Más que un valor real de biomasa es un parámetro para comparar entre árboles en función de un atributo que es relevante para la utilización de los mismos. Atendiendo a que en chañar es frecuente el hábito simpódico (árboles con varios fustes o vástagos), se usó como diámetro para calcular el $\mathrm{D}^{2} \mathrm{H}$ el DAP equivalente. Este corresponde al diámetro de la superficie circular determinada por la sumatoria de las superficies circulares asociadas a cada vástago del árbol en evaluación. Como altura se utilizó la altura total medida en terreno.

- Proyección de copa (PC): Corresponde a la superficie $\left(\mathrm{m}^{2}\right)$ de la proyección de copa, calculada en función de los diámetros de copa medidos en terreno. Es un indicador del tamaño de la misma, que resulta de interés para comparar y seleccionar individuos en función del tamaño de sus copas, como referente de la cantidad de frutos que pueden producir.

- $\quad$ Productividad de frutos (PF): Corresponde al peso seco $(\mathrm{kg})$ de la totalidad de frutos existentes en cada árbol al momento de su evaluación. 
En cuanto a la estimación de las ganancias genéticas esperadas, estas se calcularon para las mismas tres variables anteriores $\left(D^{2} \mathrm{H}, \mathrm{PC}\right.$ y $\left.\mathrm{PF}\right)$.

Para cada característica la cantidad de variación poblacional y su heredabilidad son fundamentales para estimar la ganancia genética que puede obtenerse por selección.

Al seleccionar individuos superiores en una población se produce una diferencia entre el promedio general $\left(\dot{\mathrm{X}}_{0}\right)$ y el promedio de la subpoblación seleccionada $\left(\dot{\mathrm{X}}_{\mathrm{P}}\right)$. Esta diferencia corresponde al denominado Diferencial de Selección, normalmente simbolizado por "S" (Figura Nº 1).

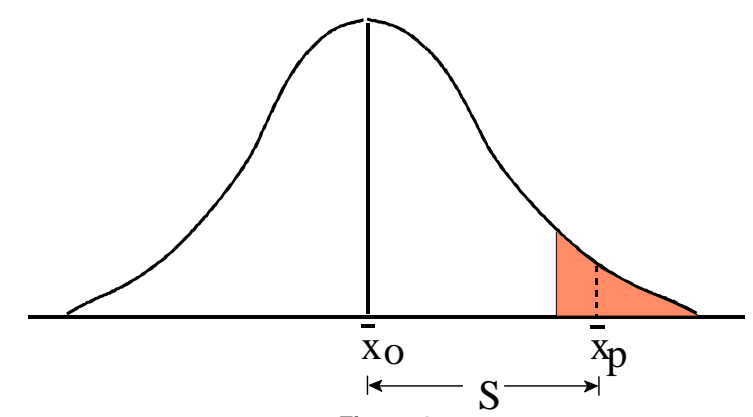

Figura 1

REPRESENTACIÓN GRÁFICA DEL DIFERENCIAL DE SELECCIÓN

Como cada característica tiene una determinada heredabilidad $\left(\mathrm{h}^{2}\right)$, no todo el diferencial de selección es traspasado a la descendencia. La heredabilidad en sentido estricto es el parámetro que determina qué proporción de la variación es transmisible a la descendencia por vía sexual, de modo que el producto entre ella y el diferencial de selección determina la Ganancia Genética Esperada, en una expresión conocida como ecuación del mejorador (1).

\section{(1) $\mathrm{GGE}=\mathrm{S}^{*} \mathrm{~h}^{2}$}

Considerando que el Diferencial de Selección (S) también puede expresarse en función de la desviación estándar fenotípica de la población $\left(\sigma_{p}\right)$ y de la intensidad de selección (i), a través de la expresión (2), entonces la fórmula de la ganancia genética esperada se hace equivalente a la expresión (3), que es la forma más común de expresarla.

(2) $S=i^{*} \sigma_{p}$

(3) $\mathrm{GGE}=\mathrm{i}^{*} \sigma_{\mathrm{p}}{ }^{*} \mathbf{h}^{2}$

Debido a las características de la distribución normal de la población, existe una relación entre la intensidad de selección (i) y la proporción de los individuos seleccionados dentro de una población.

Así, conociendo la proporción de individuos seleccionados (por ejemplo un árbol cada tantas hectáreas a un espaciamiento determinado) se puede consultar en tablas el área bajo la curva normal y obtener el valor de la intensidad de selección (i). 
Tal propiedad hace que la expresión (3) sea especialmente versátil, pues conociendo la desviación estándar fenotípica poblacional (parámetro que puede obtenerse por aplicación de técnicas de muestreo), se puede calcular la ganancia esperada asociada a distintas intensidades de selección, antes de que tal selección se efectúe.

Alternativamente, la intensidad de selección puede variarse para determinar cuántos individuos se deben seleccionar para obtener una ganancia determinada.

Para efectos de determinar las ganancia genética esperada, en este estudio se hizo uso de la ecuación del mejorador (expresión 1) y atendiendo a que no se cuenta con estimaciones de heredabilidad para las variables evaluadas, se hizo una sensibilización de este parámetro asumiendo tres escenarios probables de heredabilidad: alta $\left(h^{2}=0,30\right)$, media $\left(h^{2}=0,15\right)$ y baja $\left(h^{2}\right.$ $=0,05$ ).

\section{RESULTADOS}

Las características generales de los árboles evaluados se presentan en el Cuadro $\mathrm{N}^{\circ} 1$, donde se detallan parámetros de estadística descriptiva para las variables relevantes involucradas en los análisis; a su vez estos parámetros se desglosan por localidad y total para cada una de las variables descritas.

Los mayores valores diamétricos, se encuentran en los árboles de la localidad de Valle Fértil, que a su vez es la que manifiesta el mayor número de vástagos por árbol y proyección de copa, sin embargo y en contra de lo esperado, presenta la menor productividad promedio de frutos.

\section{ESTADÍSTICAS DESCRIPTIVA DE LAS VARIABLES CONSIDERADAS EN LA EVALUACIÓN DE ÁRBOLES SELECTOS DE CHAÑAR}

\begin{tabular}{|c|c|c|c|c|c|c|c|}
\hline Variable & Localidad & $\begin{array}{c}\text { Árboles } \\
\left(N^{\circ}\right)\end{array}$ & $\begin{array}{c}\text { Valor } \\
\text { Promedio }\end{array}$ & $\begin{array}{c}\text { Valor } \\
\text { Máximo }\end{array}$ & $\begin{array}{c}\text { Valor } \\
\text { Mínimo }\end{array}$ & $\begin{array}{l}\text { Desviación } \\
\text { Estándar }\end{array}$ & $\begin{array}{c}\text { Coeficiente } \\
\text { de Variación } \\
(\%)\end{array}$ \\
\hline \multirow{4}{*}{$\begin{array}{c}\text { HT } \\
\text { Altura Total } \\
(\mathrm{m})\end{array}$} & Piedra Colgada & 34 & 4,63 & 11,90 & 1,79 & 1,94 & 42,00 \\
\hline & Valle Fértil & 36 & 4,00 & 6,50 & 1,40 & 1,22 & 30,43 \\
\hline & San Camilo & 6 & 3,72 & 4,76 & 3,05 & 0,59 & 15,76 \\
\hline & Total & 76 & 4,26 & 11,90 & 1,40 & 1,58 & 37,08 \\
\hline \multirow{4}{*}{$\begin{array}{c}\mathrm{N}^{\circ} \text { de } \\
\text { Vástagos }\end{array}$} & Piedra Colgada & 34 & 2,94 & 7 & 1 & 1,43 & 48,76 \\
\hline & Valle Fértil & 36 & 4,75 & 16 & 1 & 3,62 & 76,22 \\
\hline & San Camilo & 6 & 2,50 & 4 & 1 & 1,05 & 41,95 \\
\hline & Total & 76 & 3,76 & 16 & 1 & 2,83 & 75,14 \\
\hline \multirow{4}{*}{$\begin{array}{c}\text { DAP } \\
\text { Equivalente } \\
(\mathrm{cm})\end{array}$} & Piedra Colgada & 34 & 17,38 & 59,00 & 5,00 & 9,98 & 57,39 \\
\hline & Valle Fértil & 36 & 24,01 & 38,88 & 5,50 & 9,05 & 37,68 \\
\hline & San Camilo & 6 & 20,13 & 27,46 & 15,00 & 5,33 & 26,46 \\
\hline & Total & 76 & 20,74 & 59,00 & 5,00 & 9,70 & 46,78 \\
\hline \multirow{4}{*}{$\begin{array}{l}\mathbf{D}^{2} \mathbf{H} \\
\left(\mathrm{m}^{3}\right)\end{array}$} & Piedra Colgada & 34 & 0,2854 & 4,1424 & 0,0060 & 0,72 & 252,51 \\
\hline & Valle Fértil & 36 & 0,2985 & 0,7089 & 0,0058 & 0,23 & 76,61 \\
\hline & San Camilo & 6 & 0,1681 & 0,3295 & 0,0815 & 0,11 & 67,41 \\
\hline & Total & 76 & 0,2823 & 4,1424 & 0,0058 & 0,50 & 178,83 \\
\hline \multirow{4}{*}{$\begin{array}{c}\text { PC } \\
\text { Proyección de } \\
\text { Copa } \\
\left(\mathrm{m}^{2}\right)\end{array}$} & Piedra Colgada & 34 & 13,65 & 83,32 & 3,03 & 14,26 & 104,49 \\
\hline & Valle Fértil & 36 & 27,83 & 87,91 & 2,50 & 19,47 & 69,95 \\
\hline & San Camilo & 6 & 24,31 & 36,53 & 11,16 & 9,43 & 38,79 \\
\hline & Total & 76 & 21,21 & 87,91 & 2,50 & 17,89 & 84,35 \\
\hline \multirow{4}{*}{$\begin{array}{c}\text { PF } \\
\text { Producción de } \\
\text { Frutos } \\
\text { (kg/árbol) }\end{array}$} & Piedra Colgada & 34 & 2,15 & 4,80 & 0,11 & 1,14 & 52,96 \\
\hline & Valle Fértil & 36 & 1,85 & 3,90 & 0,59 & 0,87 & 46,86 \\
\hline & San Camilo & 6 & 5,21 & 6,10 & 3,20 & 1,04 & 19,94 \\
\hline & Total & 76 & 2,25 & 6,10 & 0,11 & 1,33 & 59,26 \\
\hline
\end{tabular}


La mayor altura promedio la exhiben los árboles de la localidad de Piedra Colgada, la que muestra valores intermedios entre las dos localidades restantes en términos de Número de Vástagos, $D^{2} \mathrm{H}$ y Producción de Frutos; así como los menores valores medios de DAP Equivalente y Proyección de Copa. Por su parte, la mejor productividad de frutos se registra en los árboles de la localidad de San Camilo.

Respecto del comportamiento general de los árboles selectos, se destaca la escasa relación existente entre la productividad de frutos y la envergadura de copa representada por la proyección de la misma. Tal Situación se refleja en la Figura $N^{\circ} 2$, donde se grafican los valores promedios de Proyección de Copa y de Productividad de Frutos por árbol en cada localidad (para efectos de usar la misma escala, el valor de productividad de fruto está amplificado X 5). La misma situación se confirma en la Figura $\mathrm{N}^{\circ} 3$, donde se visualiza la relación entre ambas variables y su reducido coeficiente de determinación $R^{2}=0,03$

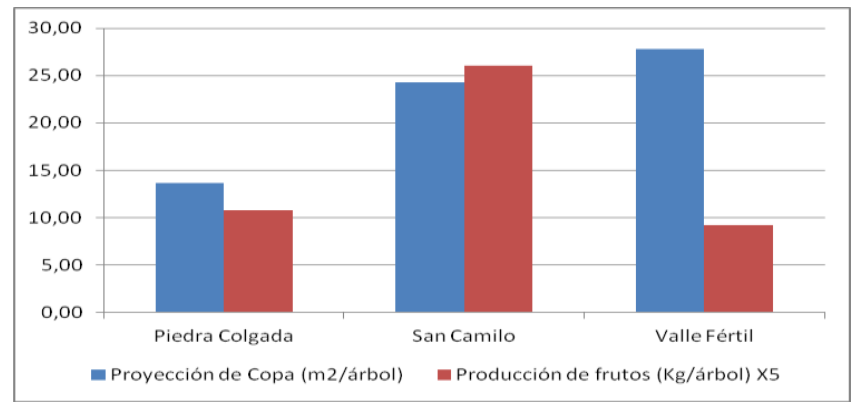

Figura $\mathrm{N}^{\circ} 2$

VALORES MEDIOS DE PROYECCIÓN DE COPA Y PRODUCTIVIDAD DE FRUTOS POR LOCALIDAD

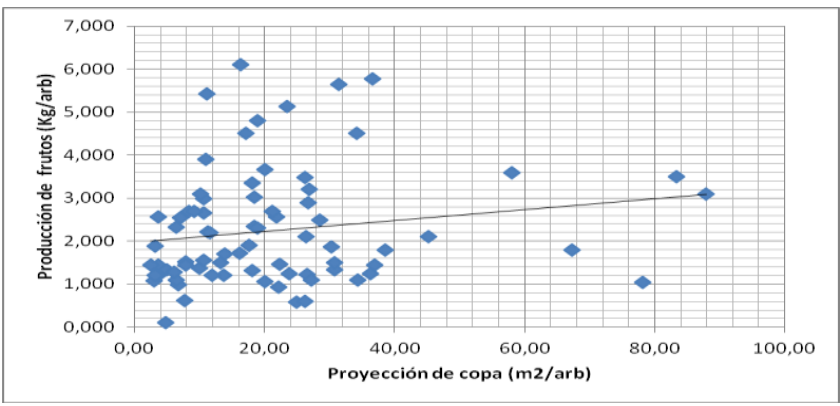

Figura $\mathrm{N}^{\circ} 3$

RELACIÓN ENTRE PROYECCIÓN DE COPA Y PRODUCTIVIDAD DE FRUTOS A NIVEL DE ÁRBOLES INDIVIDUALES

Un segundo factor a resaltar en los árboles analizados es la alta variabilidad que presentan las variables evaluadas, tanto dentro de las localidades como a nivel general. Esta situación queda reflejada en los parámetros de dispersión del Cuadro $N^{\circ} 1$ (valores máximos y mínimos, desviación estándar y coeficiente de variación) y corresponde a un escenario esperado al realizar selección masal en rodales naturales, donde no hay garantías de coetaneidad de los árboles. Por lo mismo, las diferencias de edad entre ellos agregan variabilidad que dificulta la selección. 
En cuanto a la estimación del porcentaje de superioridad fenotípica y de las ganancias genéticas esperadas, los resultados se presentan en el Cuadro $\mathrm{N}^{\circ} 2$. En el cuadro se presenta un ranking con los 76 árboles evaluados, ordenados en función de la superioridad combinada manifestada en $D^{2} \mathrm{H}$, Proyección de Copa y Productividad de Frutos, la que se obtuvo como promedio simple de las superioridades expresadas en cada una de las variables anteriores en forma individual. En el mismo cuadro se agregan, para cada árbol, las ganancias genéticas esperadas para cada una de las variables en evaluación en los escenarios de heredabilidad media y baja.

En esta evaluación se destaca como primer árbol del ranking, al individuo $\mathrm{N}^{\circ} 3$ del cuartel $N^{\circ} 3$ de la localidad de Valle Fértil, el cual manifiesta un porcentaje de superioridad fenotípica considerablemente mayor al de todos los individuos restantes $(232,1 \%)$. Este alto valor se deriva fundamentalmente del efecto de su gran envergadura (y posiblemente mayor edad) en relación a sus árboles de comparación, lo cual tiene una gran incidencia sobre la superioridad expresada en el $D^{2} \mathrm{H}$ y la Proyección de Copa. Existen 28 árboles con porcentajes de superioridad fenotípica mayor que cero, de los cuales 15 expresan valores conjuntos superiores al $50 \%$.

A nivel de variables individuales no existe necesariamente coincidencia en la magnitud de la superioridad. Es decir, árboles que se destacan en $D^{2} \mathrm{H}$ no necesariamente lo hacen en productividad de frutos. En efecto, el segundo árbol del ranking por su valor combinado, presenta altos niveles de superioridad en $D^{2} \mathrm{H}$ y Proyección de Copa, pero un valor negativo en Producción de Frutos.

Las situaciones inversas o recíprocas también son comunes. Por lo mismo, aunque solo 15 árboles presentan superioridad combinada mayor a $50 \%$, hay numerosos otros individuos que aún con baja superioridad combinada presentan, a nivel de variables independientes, porcentajes de superioridad interesantes que se reflejan en estimativas de ganancia genética que aconsejan su consideración.

En tal sentido la información generada resulta de valor para apoyar la selección de los árboles que se incorporen a un programa de mejoramiento genético, por cuanto permite hacer otros ordenamientos en función de cada variable, para seleccionar líneas de mejora independientes para biomasa o producción de frutos, o implementar otros criterios de selección.

Por ejemplo, se puede priorizar a los árboles de mayor productividad de frutos y posteriormente, entre ellos, seleccionar en tándem en función de $D^{2} H$. Esa y todas las demás combinaciones posibles pueden implementarse en función de los datos presentados en el Cuadro $\mathrm{N}^{\circ} 2$.

Cuadro $\mathrm{N}^{\circ} 2$

SUPERIORIDAD FENOTÍPICA EN D²H, PROYECCIÓN DE COPA (PC) Y PRODUCTIVIDAD DE FRUTOS (PF), ORDENADA EN FUNCIÓN DE LA SUPERIORIDAD COMBINADA PARA LAS TRES VARIABLES

\begin{tabular}{|c|c|c|c|c|c|c|c|c|c|c|}
\hline \multirow[t]{2}{*}{ Ranking } & \multicolumn{3}{|c|}{$\begin{array}{l}\text { Identificación } \\
\text { Candidato }\end{array}$} & \multicolumn{3}{|c|}{ Variables } & \multicolumn{4}{|c|}{$\begin{array}{c}\text { Superioridad Fenotípica } \\
(\%)\end{array}$} \\
\hline & Sector-Cua & el-Á & $\mathrm{Ol}^{(1)}$ & $\begin{array}{l}D^{2} H \\
\left(m^{3}\right)\end{array}$ & $\begin{array}{c}\mathrm{PC} \\
\left(\mathrm{m}^{2}\right)\end{array}$ & $\begin{array}{c}\text { PF } \\
\text { (Kg/árbol) }\end{array}$ & $\begin{array}{l}D^{2} H \\
\left(m^{3}\right)\end{array}$ & $\begin{array}{l}\mathrm{PC} \\
\left(\mathrm{m}^{2}\right)\end{array}$ & $\begin{array}{c}\text { PF } \\
\text { (Kg/árbol) }\end{array}$ & Comb. \\
\hline 1 & V. Fértil & 3 & $3-0$ & 0,7089 & 87,91 & 3,100 & 347,8 & 324,4 & $\begin{array}{r}24,0 \\
\end{array}$ & 232,1 \\
\hline 2 & P. Colgada & 6 & $6-0$ & 4,1424 & 83,32 & 3,500 & 168,7 & 130,5 & $-9,7$ & 96,5 \\
\hline 3 & V. Fértil & 4 & $4-0$ & 0,3361 & 45,19 & 2,100 & 112,8 & 87,0 & 77,7 & 92,5 \\
\hline 4 & V. Fértil & 1 & $1-0$ & 0,2256 & 26,28 & 3,480 & 97,3 & 64,1 & 74,3 & 78,5 \\
\hline 5 & P. Colgada & 5 & $5-0$ & 0,3246 & 20,07 & 3,660 & 116,3 & 70,3 & 45,3 & 77,3 \\
\hline 6 & P. Colgada & 2 & $2-0$ & 0,2885 & 21,81 & 2,570 & 92,1 & 73,8 & 57,4 & 74,4 \\
\hline 7 & P. Colgada & 4 & $4-0$ & 0,1660 & 16,12 & 1,715 & 140,3 & 60,0 & $-8,8$ & 63,9 \\
\hline 8 & P. Colgada & 5 & $5-4$ & 0,2489 & 17,09 & 4,500 & 65,9 & 45,0 & 78,7 & 63,2 \\
\hline 9 & P. Colgada & 2 & $2-5$ & 0,3807 & 22,10 & 0,925 & 153,4 & 76,1 & $-43,3$ & 62,1 \\
\hline 10 & V. Fértil & 6 & $6-2$ & 0,7000 & 58,09 & 3,600 & 57,5 & 66,7 & 50,0 & 58,1 \\
\hline 11 & P. Colgada & 3 & $3-0$ & 0,2334 & 21,24 & 2,690 & 77,3 & 86,8 & 8,0 & 57,3 \\
\hline 12 & P. Colgada & 4 & $4-1$ & 0,0847 & 18,40 & 3,025 & 22,6 & 82,7 & 60,9 & 55,4 \\
\hline
\end{tabular}




\begin{tabular}{|c|c|c|c|c|c|c|c|c|c|c|}
\hline \multirow[t]{2}{*}{ Ranking } & \multirow{2}{*}{\multicolumn{3}{|c|}{$\begin{array}{c}\begin{array}{c}\text { Identificación } \\
\text { Candidato }\end{array} \\
\text { Sector-Cuartel-Árbol }^{(1)}\end{array}$}} & \multicolumn{3}{|c|}{ Variables } & \multicolumn{4}{|c|}{$\begin{array}{l}\text { Superioridad Fenotípica } \\
(\%)\end{array}$} \\
\hline & & & & $\begin{array}{l}D^{2} H \\
\left(m^{3}\right)\end{array}$ & $\begin{array}{l}\mathrm{PC} \\
\left(\mathrm{m}^{2}\right)\end{array}$ & \begin{tabular}{|c|} 
PF \\
(Kg/árbol)
\end{tabular} & $\begin{array}{l}D^{2} \mathrm{H} \\
\left(\mathrm{m}^{3}\right)\end{array}$ & $\begin{array}{c}\mathrm{PC} \\
\left(\mathrm{m}^{2}\right)\end{array}$ & $\begin{array}{c}\text { PF } \\
\text { (Kg/árbol) }\end{array}$ & Comb. \\
\hline 13 & V. Fértil & 2 & $2-1$ & 0,6592 & 78,15 & 1,050 & 89,4 & 97,9 & $-21,4$ & 55,3 \\
\hline 14 & P. Colgada & 1 & $1-1$ & 0,1752 & 9,93 & 1,365 & 98,7 & 33,3 & 25,6 & 52,5 \\
\hline 15 & S. Camilo & 1 & $1-3$ & 0,3295 & 36,53 & 5,780 & 96,0 & 50,3 & 10,9 & 52,4 \\
\hline 16 & V. Fértil & 5 & $5-5$ & 0,5901 & 67,20 & 1,800 & 3,9 & 111,6 & 7,4 & 41,0 \\
\hline 17 & P. Colgada & 2 & $2-2$ & 0,1894 & 18,44 & 2,350 & 26,1 & 46,9 & 44,0 & 39,0 \\
\hline 18 & S. Camilo & 1 & $1-5$ & 0,2971 & 31,42 & 5,650 & 76,7 & 29,3 & 8,4 & 38,1 \\
\hline 19 & V. Fértil & 1 & $1-4$ & 0,2183 & 17,53 & 1,900 & 90,9 & 9,4 & $-4,8$ & 31,8 \\
\hline 20 & P. Colgada & 1 & $1-0$ & 0,1105 & 7,92 & 1,525 & 25,3 & 6,3 & 40,3 & 24,0 \\
\hline 21 & V. Fértil & 5 & $5-1$ & 0,5252 & 18,10 & 3,360 & $-7,5$ & $-43,0$ & 100,4 & 16,6 \\
\hline 22 & P. Colgada & 3 & $3-2$ & 0,1986 & 11,34 & 2,210 & 50,8 & $-0,3$ & $-11,3$ & 13,1 \\
\hline 23 & P. Colgada & 1 & $1-5$ & 0,0390 & 10,61 & 1,560 & $-55,8$ & 42,4 & 43,5 & 10,1 \\
\hline 24 & V. Fértil & 2 & $2-5$ & 0,4861 & 30,68 & 1,340 & 39,7 & $-22,3$ & 0,2 & 5,9 \\
\hline 25 & P. Colgada & 4 & $4-3$ & 0,0776 & 13,72 & 1,200 & 12,3 & 36,3 & $-36,2$ & 4,1 \\
\hline 26 & V. Fértil & 6 & $6-0$ & 0,5498 & 38,48 & 1,800 & 23,7 & 10,5 & $-25,0$ & 3,1 \\
\hline 27 & V. Fértil & 4 & $4-2$ & 0,2361 & 26,28 & 0,600 & 49,5 & 8,8 & $-49,2$ & 3,0 \\
\hline 28 & P. Colgada & 3 & $3-1$ & 0,1264 & 10,61 & 2,980 & $-4,0$ & $-6,7$ & 19,6 & 3,0 \\
\hline 29 & V. Fértil & 4 & $4-5$ & 0,1309 & 22,40 & 1,460 & $-17,1$ & $-7,3$ & 23,6 & $-0,3$ \\
\hline 30 & V. Fértil & 1 & $1-3$ & 0,0748 & 18,90 & 2,300 & $-34,6$ & 17,9 & 15,2 & $-0,5$ \\
\hline 31 & P. Colgada & 6 & $6-2$ & 1,3429 & 34,11 & 4,500 & $-12,9$ & $-5,7$ & 16,1 & $-0,8$ \\
\hline 32 & V. Fértil & 2 & $2-0$ & 0,2568 & 30,34 & 1,860 & $-26,2$ & $-23,2$ & 39,2 & $-3,4$ \\
\hline 33 & V. Fértil & 5 & $5-0$ & 0,5467 & 36,32 & 1,240 & $-3,7$ & 14,3 & $-26,0$ & $-5,1$ \\
\hline 34 & V. Fértil & 2 & $2-3$ & 0,2846 & 36,85 & 1,450 & $-18,2$ & $-6,7$ & 8,5 & $-5,5$ \\
\hline 35 & P. Colgada & 5 & $5-3$ & 0,1057 & 10,15 & 3,100 & $-29,5$ & $-13,9$ & 23,1 & $-6,8$ \\
\hline 36 & V. Fértil & 5 & $5-4$ & 0,6983 & 27,11 & 1,100 & 23,0 & $-14,7$ & $-34,4$ & $-8,7$ \\
\hline 37 & V. Fértil & 1 & $1-2$ & 0,1137 & 13,85 & 1,700 & $-0,6$ & $-13,5$ & $-14,9$ & $-9,6$ \\
\hline 38 & V. Fértil & 6 & $6-3$ & 0,4411 & 26,42 & 2,100 & $-0,8$ & $-24,2$ & $-12,5$ & $-12,5$ \\
\hline 39 & V. Fértil & 6 & $6-1$ & 0,3360 & 28,56 & 2,500 & $-24,4$ & $-18,0$ & 4,2 & $-12,8$ \\
\hline 40 & S. Camilo & 1 & $1-4$ & 0,1092 & 23,50 & 5,125 & $-35,0$ & $-3,3$ & $-1,7$ & $-13,3$ \\
\hline 41 & P. Colgada & 3 & $3-4$ & 0,0905 & 11,61 & 2,200 & $-31,3$ & 2,1 & $-11,7$ & $-13,6$ \\
\hline 42 & V. Fértil & 4 & $4-3$ & 0,1322 & 20,07 & 1,060 & $-16,3$ & $-16,9$ & $-10,3$ & $-14,5$ \\
\hline 43 & V. Fértil & 6 & $6-4$ & 0,4248 & 30,83 & 1,500 & $-4,4$ & $-11,5$ & $-37,5$ & $-17,8$ \\
\hline 44 & V. Fértil & 6 & $6-5$ & 0,2153 & 26,65 & 2,900 & $-51,6$ & $-23,5$ & 20,8 & $-18,1$ \\
\hline 45 & S. Camilo & 1 & $1-0$ & 0,1031 & 16,30 & 6,100 & $-38,7$ & $-33,0$ & 17,0 & $-18,2$ \\
\hline 46 & V. Fértil & 5 & $5-3$ & 0,5483 & 23,76 & 1,240 & $-3,4$ & $-25,2$ & $-26,0$ & $-18,2$ \\
\hline 47 & V. Fértil & 3 & $3-5$ & 0,0569 & 10,93 & 3,900 & $-64,1$ & $-47,3$ & 56,0 & $-18,4$ \\
\hline 48 & P. Colgada & 5 & $5-1$ & 0,1342 & 12,01 & 1,200 & $-10,6$ & 1,9 & $-52,3$ & $-20,4$ \\
\hline 49 & V. Fértil & 3 & $3-1$ & 0,1224 & 10,69 & 2,650 & $-22,7$ & $-48,4$ & 6,0 & $-21,7$ \\
\hline 50 & V. Fértil & 2 & $2-2$ & 0,2272 & 34,37 & 1,100 & $-34,7$ & $-13,0$ & $-17,7$ & $-21,8$ \\
\hline 51 & P. Colgada & 1 & $1-2$ & 0,1351 & 4,73 & 0,111 & 53,2 & $-36,4$ & $-89,8$ & $-24,3$ \\
\hline 52 & P. Colgada & 3 & $3-5$ & 0,0985 & 6,38 & 2,325 & $-25,2$ & $-43,9$ & $-6,7$ & $-25,3$ \\
\hline 53 & V. Fértil & 5 & $5-2$ & 0,4974 & 18,10 & 1,320 & $-12,4$ & $-43,0$ & $-21,3$ & $-25,6$ \\
\hline 54 & S. Camilo & 1 & $1-1$ & 0,0815 & 26,92 & 3,200 & $-51,6$ & 10,8 & $-38,6$ & $-26,5$ \\
\hline 55 & P. Colgada & 4 & $4-2$ & 0,0324 & 3,60 & 2,560 & $-53,0$ & $-64,3$ & 36,2 & $-27,0$ \\
\hline 56 & P. Colgada & 1 & $1-4$ & 0,0473 & 7,65 & 0,615 & $-46,4$ & 2,7 & $-43,4$ & $-29,0$ \\
\hline 57 & V. Fértil & 2 & $2-4$ & 0,1740 & 26,56 & 1,220 & $-50,0$ & $-32,7$ & $-8,7$ & $-30,5$ \\
\hline 58 & S. Camilo & 1 & $1-2$ & 0,0885 & 11,16 & 5,420 & $-47,4$ & $-54,1$ & 4,0 & $-32,5$ \\
\hline 59 & P. Colgada & 6 & $6-1$ & 0,3785 & 18,90 & 4,800 & $-75,5$ & $-47,7$ & 23,9 & $-33,1$ \\
\hline 60 & P. Colgada & 1 & $1-3$ & 0,0220 & 3,85 & 1,345 & $-75,0$ & $-48,3$ & 23,8 & $-33,2$ \\
\hline 61 & V. Fértil & 1 & $1-1$ & 0,0480 & 13,20 & 1,500 & $-58,0$ & $-17,6$ & $-24,9$ & $-33,5$ \\
\hline 62 & V. Fértil & 4 & $4-1$ & 0,0733 & 24,98 & 0,585 & $-53,6$ & 3,4 & $-50,5$ & $-33,6$ \\
\hline 63 & P. Colgada & 3 & $3-3$ & 0,0426 & 7,05 & 2,545 & $-67,6$ & $-38,0$ & 2,1 & $-34,5$ \\
\hline 64 & P. Colgada & 5 & $5-2$ & 0,0746 & 7,79 & 1,450 & $-50,3$ & $-33,9$ & $-42,4$ & $-42,2$ \\
\hline 65 & V. Fértil & 3 & $3-3$ & 0,0275 & 9,11 & 2,700 & $-82,6$ & $-56,0$ & 8,0 & $-43,6$ \\
\hline 66 & P. Colgada & 4 & $4-5$ & 0,0267 & 5,01 & 1,330 & $-61,3$ & $-50,3$ & $-29,3$ & $-47,0$ \\
\hline 67 & V. Fértil & 4 & $4-4$ & 0,0390 & 6,07 & 1,285 & $-75,3$ & $-74,9$ & 8,7 & $-47,1$ \\
\hline 68 & P. Colgada & 2 & $2-3$ & 0,0181 & 3,16 & 1,890 & $-87,9$ & $-74,8$ & 15,8 & $-49,0$ \\
\hline 69 & P. Colgada & 4 & $4-4$ & 0,0270 & 3,58 & 1,450 & $-60,9$ & $-64,5$ & $-22,9$ & $-49,4$ \\
\hline 70 & P. Colgada & 2 & $2-4$ & 0,0185 & 6,77 & 0,980 & $-87,7$ & $-46,1$ & $-40,0$ & $-57,9$ \\
\hline
\end{tabular}




\begin{tabular}{|c|c|c|c|c|c|c|c|c|c|c|}
\hline \multirow[t]{2}{*}{ Ranking } & \multirow{2}{*}{\multicolumn{3}{|c|}{$\begin{array}{c}\begin{array}{c}\text { Identificación } \\
\text { Candidato }\end{array} \\
\text { ector-Cuartel-Árbol }{ }^{(1)}\end{array}$}} & \multicolumn{3}{|c|}{ Variables } & \multicolumn{4}{|c|}{$\begin{array}{l}\text { Superioridad Fenotípica } \\
(\%)\end{array}$} \\
\hline & & & & $\begin{array}{l}D^{2} H \\
\left(m^{3}\right)\end{array}$ & $\begin{array}{l}\mathrm{PC} \\
\left(\mathrm{m}^{2}\right)\end{array}$ & \begin{tabular}{|c} 
PF \\
(Kg/árbol)
\end{tabular} & $\begin{array}{l}D^{2} \mathrm{H} \\
\left(m^{3}\right)\end{array}$ & $\begin{array}{l}\mathrm{PC} \\
\left(\mathrm{m}^{2}\right)\end{array}$ & $\begin{array}{c}\text { PF } \\
\text { (Kg/árbol) }\end{array}$ & Comb. \\
\hline 71 & P. Colgada & 6 & $6-3$ & 0,3035 & 8,30 & 2,700 & $-80,3$ & $-77,1$ & $-30,3$ & $-62,6$ \\
\hline 72 & V. Fértil & 1 & $1-5$ & 0,0058 & 6,36 & 1,100 & $-94,9$ & $-60,3$ & $-44,9$ & $-66,7$ \\
\hline 73 & P. Colgada & 2 & $2-1$ & 0,0060 & 3,03 & 1,080 & $-96,0$ & $-75,8$ & $-33,8$ & $-68,6$ \\
\hline 74 & P. Colgada & 5 & $5-5$ & 0,0124 & 3,60 & 1,200 & $-91,7$ & $-69,5$ & $-52,3$ & $-71,2$ \\
\hline 75 & V. Fértil & 3 & $3-2$ & 0,0261 & 3,16 & 1,200 & $-83,5$ & $-84,8$ & $-52,0$ & $-73,4$ \\
\hline 76 & V. Fértil & 3 & $3-4$ & 0,0080 & 2,50 & 1,450 & $-95,0$ & $-87,9$ & $-42,0$ & $-75,0$ \\
\hline
\end{tabular}

(1) Interpretación del ranking: Ej, Sector Piedra Colgada, Cuartel 5, árbol seleccionado $\mathrm{N}^{\circ} 5$ se ubica en lugar 5 del ranking y árbol acompañante $\mathrm{N}^{\circ} 4$ del mismo árbol seleccionado $\mathrm{N}^{\circ} 5$ se ubica en lugar 8 del ranking.

Cuadro $\mathrm{N}^{\circ} 3$

GANANCIAS GENÉTICAS ESPERADAS EN DOS ESCENARIOS DE HEREDABILIDAD, ORDENADO EN FUNCIÓN DE LA SUPERIORIDAD FENOTÍPICA COMBINADA DEL CUADRO ANTERIOR

\begin{tabular}{|c|c|c|c|c|c|c|c|c|c|}
\hline \multirow{3}{*}{ Ranking } & \multirow{2}{*}{\multicolumn{3}{|c|}{ Identificación Candidato }} & \multicolumn{6}{|c|}{$\begin{array}{c}\text { Ganancias Genéticas Esperadas } \\
(\%)\end{array}$} \\
\hline & & & & \multicolumn{3}{|c|}{$\left(h^{2}=0,05\right)$} & \multicolumn{3}{|c|}{$\left(h^{2}=0,15\right)$} \\
\hline & \multicolumn{3}{|c|}{ Sector-Cuartel-Árbol } & $\begin{array}{l}D^{2} \mathrm{H} \\
\left(m^{3}\right)\end{array}$ & $\begin{array}{c}P C \\
\left(\mathrm{~m}^{2}\right)\end{array}$ & $\begin{array}{c}\text { PF } \\
\text { (Kg/árbol) }\end{array}$ & $\begin{array}{l}D^{2} \mathrm{H} \\
\left(m^{3}\right)\end{array}$ & $\begin{array}{l}\mathrm{PC} \\
\left(\mathrm{m}^{2}\right)\end{array}$ & $\begin{array}{c}\text { PF } \\
\text { (Kg/árbol) }\end{array}$ \\
\hline 1 & V. Fértil & 3 & $3-0$ & 17,39 & 16,22 & 1,20 & 52,18 & 48,65 & 3,60 \\
\hline 2 & P. Colgada & 6 & $6-0$ & 8,43 & 6,52 & $-0,48$ & 25,30 & 19,57 & $-1,45$ \\
\hline 3 & V. Fértil & 4 & $4-0$ & 5,64 & 4,35 & 3,89 & 16,92 & 13,05 & 11,66 \\
\hline 4 & V. Fértil & 1 & $1-0$ & 4,86 & 3,20 & 3,71 & 14,59 & 9,61 & 11,14 \\
\hline 5 & P. Colgada & 5 & $5-0$ & 5,81 & 3,51 & 2,27 & 17,44 & 10,54 & 6,80 \\
\hline 6 & P. Colgada & 2 & $2-0$ & 4,60 & 3,69 & 2,87 & 13,81 & 11,07 & 8,61 \\
\hline 7 & P. Colgada & 4 & $4-0$ & 7,02 & 3,00 & $-0,44$ & 21,05 & 9,01 & $-1,32$ \\
\hline 8 & P. Colgada & 5 & $5-4$ & 3,29 & 2,25 & 3,93 & 9,88 & 6,76 & 11,80 \\
\hline 9 & P. Colgada & 2 & $2-5$ & 7,67 & 3,81 & $-2,17$ & 23,01 & 11,42 & $-6,50$ \\
\hline 10 & V. Fértil & 6 & $6-2$ & 2,87 & 3,34 & 2,50 & 8,62 & 10,01 & 7,50 \\
\hline 11 & P. Colgada & 3 & $3-0$ & 3,86 & 4,34 & 0,40 & 11,59 & 13,02 & 1,19 \\
\hline 12 & P. Colgada & 4 & $4-1$ & 1,13 & 4,13 & 3,05 & 3,40 & 12,40 & 9,14 \\
\hline 13 & V. Fértil & 2 & $2-1$ & 4,47 & 4,89 & $-1,07$ & 13,41 & 14,68 & $-3,22$ \\
\hline 14 & P. Colgada & 1 & $1-1$ & 4,94 & 1,66 & 1,28 & 14,81 & 4,99 & 3,84 \\
\hline 15 & S. Camilo & 1 & $1-3$ & 4,80 & 2,51 & 0,54 & 14,40 & 7,54 & 1,63 \\
\hline 16 & V. Fértil & 5 & $5-5$ & 0,20 & 5,58 & 0,37 & 0,59 & 16,74 & 1,10 \\
\hline 17 & P. Colgada & 2 & $2-2$ & 1,31 & 2,34 & 2,20 & 3,92 & 7,03 & 6,59 \\
\hline 18 & S. Camilo & 1 & $1-5$ & 3,83 & 1,46 & 0,42 & 11,50 & 4,39 & 1,26 \\
\hline 19 & V. Fértil & 1 & $1-4$ & 4,54 & 0,47 & $-0,24$ & 13,63 & 1,42 & $-0,73$ \\
\hline 20 & P. Colgada & 1 & $1-0$ & 1,26 & 0,32 & 2,02 & 3,79 & 0,95 & 6,05 \\
\hline 21 & V. Fértil & 5 & $5-1$ & $-0,37$ & $-2,15$ & 5,02 & $-1,12$ & $-6,45$ & 15,06 \\
\hline 22 & P. Colgada & 3 & $3-2$ & 2,54 & $-0,01$ & $-0,57$ & 7,63 & $-0,04$ & $-1,70$ \\
\hline 23 & P. Colgada & 1 & $1-5$ & $-2,79$ & 2,12 & 2,18 & $-8,37$ & 6,37 & 6,53 \\
\hline 24 & V. Fértil & 2 & $2-5$ & 1,98 & $-1,12$ & 0,01 & 5,95 & $-3,35$ & 0,04 \\
\hline 25 & P. Colgada & 4 & $4-3$ & 0,62 & 1,81 & $-1,81$ & 1,85 & 5,44 & $-5,43$ \\
\hline 26 & V. Fértil & 6 & $6-0$ & 1,18 & 0,52 & $-1,25$ & 3,55 & 1,57 & $-3,75$ \\
\hline 27 & V. Fértil & 4 & $4-2$ & 2,48 & 0,44 & $-2,46$ & 7,43 & 1,32 & $-7,38$ \\
\hline 28 & P. Colgada & 3 & $3-1$ & $-0,20$ & $-0,34$ & 0,98 & $-0,60$ & $-1,01$ & 2,94 \\
\hline 29 & V. Fértil & 4 & $4-5$ & $-0,86$ & $-0,37$ & 1,18 & $-2,57$ & $-1,10$ & 3,53 \\
\hline 30 & V. Fértil & 1 & $1-3$ & $-1,73$ & 0,90 & 0,76 & $-5,19$ & 2,69 & 2,28 \\
\hline 31 & P. Colgada & 6 & $6-2$ & $-0,65$ & $-0,28$ & 0,81 & $-1,94$ & $-0,85$ & 2,42 \\
\hline 32 & V. Fértil & 2 & $2-0$ & $-1,31$ & $-1,16$ & 1,96 & $-3,93$ & $-3,48$ & 5,87 \\
\hline 33 & V. Fértil & 5 & $5-0$ & $-0,19$ & 0,72 & $-1,30$ & $-0,56$ & 2,15 & $-3,91$ \\
\hline 34 & V. Fértil & 2 & $2-3$ & $-0,91$ & $-0,33$ & 0,42 & $-2,73$ & $-1,00$ & 1,27 \\
\hline 35 & P. Colgada & 5 & $5-3$ & $-1,48$ & $-0,69$ & 1,15 & $-4,43$ & $-2,08$ & 3,46 \\
\hline 36 & V. Fértil & 5 & $5-4$ & 1,15 & $-0,73$ & $-1,72$ & 3,45 & $-2,20$ & $-5,16$ \\
\hline 37 & V. Fértil & 1 & $1-2$ & $-0,03$ & $-0,68$ & $-0,74$ & $-0,08$ & $-2,03$ & $-2,23$ \\
\hline 38 & V. Fértil & 6 & $6-3$ & $-0,04$ & $-1,21$ & $-0,63$ & $-0,11$ & $-3,62$ & $-1,88$ \\
\hline 39 & V. Fértil & 6 & $6-1$ & $-1,22$ & $-0,90$ & 0,21 & $-3,66$ & $-2,70$ & 0,63 \\
\hline
\end{tabular}




\begin{tabular}{|c|c|c|c|c|c|c|c|c|c|}
\hline \multirow{3}{*}{ Ranking } & \multirow{2}{*}{\multicolumn{3}{|c|}{ Identificación Candidato }} & \multicolumn{6}{|c|}{$\begin{array}{c}\text { Ganancias Genéticas Esperadas } \\
(\%)\end{array}$} \\
\hline & & & & \multicolumn{3}{|c|}{$\left(h^{2}=0,05\right)$} & \multicolumn{3}{|c|}{$\left(h^{2}=0,15\right)$} \\
\hline & \multicolumn{3}{|c|}{ Sector-Cuartel-Árbol } & $\begin{array}{l}D^{2} H \\
\left(m^{3}\right)\end{array}$ & $\left(m^{2}\right)$ & $\begin{array}{c}\text { PF } \\
\text { (Kg/árbol) }\end{array}$ & $\begin{array}{l}D^{2} H \\
\left(m^{3}\right)\end{array}$ & $\begin{array}{l}\mathrm{PC} \\
\left(\mathrm{m}^{2}\right)\end{array}$ & $\begin{array}{c}\mathrm{PF} \\
\text { (Kg/árbol) }\end{array}$ \\
\hline 40 & S. Camilo & 1 & $1-4$ & $-1,75$ & $-0,17$ & $-0,08$ & $-5,25$ & $-0,50$ & $-0,25$ \\
\hline 41 & P. Colgada & 3 & $3-4$ & $-1,56$ & 0,11 & $-0,59$ & $-4,69$ & 0,32 & $-1,76$ \\
\hline 42 & V. Fértil & 4 & $4-3$ & $-0,82$ & $-0,85$ & $-0,51$ & $-2,45$ & $-2,54$ & $-1,54$ \\
\hline 43 & V. Fértil & 6 & $6-4$ & $-0,22$ & $-0,58$ & $-1,88$ & $-0,67$ & $-1,73$ & $-5,63$ \\
\hline 44 & V. Fértil & 6 & $6-5$ & $-2,58$ & $-1,18$ & 1,04 & $-7,73$ & $-3,53$ & 3,13 \\
\hline 45 & S. Camilo & 1 & $1-0$ & $-1,93$ & $-1,65$ & 0,85 & $-5,80$ & $-4,94$ & 2,55 \\
\hline 46 & V. Fértil & 5 & $5-3$ & $-0,17$ & $-1,26$ & $-1,30$ & $-0,51$ & $-3,78$ & $-3,91$ \\
\hline 47 & V. Fértil & 3 & $3-5$ & $-3,20$ & $-2,36$ & 2,80 & $-9,61$ & $-7,09$ & 8,40 \\
\hline 48 & P. Colgada & 5 & $5-1$ & $-0,53$ & 0,09 & $-2,62$ & $-1,59$ & 0,28 & $-7,85$ \\
\hline 49 & V. Fértil & 3 & $3-1$ & $-1,13$ & $-2,42$ & 0,30 & $-3,40$ & $-7,26$ & 0,90 \\
\hline 50 & V. Fértil & 2 & $2-2$ & $-1,74$ & $-0,65$ & $-0,89$ & $-5,21$ & $-1,95$ & $-2,66$ \\
\hline 51 & P. Colgada & 1 & $1-2$ & 2,66 & $-1,82$ & $-4,49$ & 7,98 & $-5,47$ & $-13,47$ \\
\hline 52 & P. Colgada & 3 & $3-5$ & $-1,26$ & $-2,19$ & $-0,33$ & $-3,78$ & $-6,58$ & $-1,00$ \\
\hline 53 & V. Fértil & 5 & $5-2$ & $-0,62$ & $-2,15$ & $-1,06$ & $-1,86$ & $-6,45$ & $-3,19$ \\
\hline 54 & S. Camilo & 1 & $1-1$ & $-2,58$ & 0,54 & $-1,93$ & $-7,73$ & 1,62 & $-5,79$ \\
\hline 55 & P. Colgada & 4 & $4-2$ & $-2,65$ & $-3,21$ & 1,81 & $-7,95$ & $-9,64$ & 5,43 \\
\hline 56 & P. Colgada & 1 & $1-4$ & $-2,32$ & 0,13 & $-2,17$ & $-6,96$ & 0,40 & $-6,51$ \\
\hline 57 & V. Fértil & 2 & $2-4$ & $-2,50$ & $-1,64$ & $-0,44$ & $-7,50$ & $-4,91$ & $-1,31$ \\
\hline 58 & S. Camilo & 1 & $1-2$ & $-2,37$ & $-2,70$ & 0,20 & $-7,11$ & $-8,11$ & 0,60 \\
\hline 59 & P. Colgada & 6 & $6-1$ & $-3,77$ & $-2,39$ & 1,19 & $-11,32$ & $-7,16$ & 3,58 \\
\hline 60 & P. Colgada & 1 & $1-3$ & $-3,75$ & $-2,41$ & 1,19 & $-11,25$ & $-7,24$ & 3,56 \\
\hline 61 & V. Fértil & 1 & $1-1$ & $-2,90$ & $-0,88$ & $-1,24$ & $-8,70$ & $-2,64$ & $\begin{array}{l}-3,73 \\
\end{array}$ \\
\hline 62 & V. Fértil & 4 & $4-1$ & $-2,68$ & 0,17 & $-2,52$ & $-8,03$ & 0,51 & $-7,57$ \\
\hline 63 & P. Colgada & 3 & $3-3$ & $-3,38$ & $-1,90$ & 0,11 & $-10,14$ & $-5,71$ & 0,32 \\
\hline 64 & P. Colgada & 5 & $5-2$ & $-2,51$ & $-1,69$ & $-2,12$ & $-7,54$ & $-5,08$ & $-6,36$ \\
\hline 65 & V. Fértil & 3 & $3-3$ & $-4,13$ & $-2,80$ & 0,40 & $-12,40$ & $-8,41$ & 1,20 \\
\hline 66 & P. Colgada & 4 & $4-5$ & $-3,07$ & $-2,51$ & $-1,46$ & $-9,20$ & $-7,54$ & $-4,39$ \\
\hline 67 & V. Fértil & 4 & $4-4$ & $-3,77$ & $-3,74$ & 0,44 & $-11,30$ & $-11,23$ & 1,31 \\
\hline 68 & P. Colgada & 2 & $2-3$ & $-4,40$ & $-3,74$ & 0,79 & $-13,19$ & $-11,23$ & 2,37 \\
\hline 69 & P. Colgada & 4 & $4-4$ & $-3,05$ & $-3,22$ & $-1,14$ & $-9,14$ & $-9,67$ & $-3,43$ \\
\hline 70 & P. Colgada & 2 & $2-4$ & $-4,38$ & $-2,30$ & $-2,00$ & $-13,15$ & $-6,91$ & $-6,00$ \\
\hline 71 & P. Colgada & 6 & $6-3$ & $-4,02$ & $-3,85$ & $-1,52$ & $-12,05$ & $-11,56$ & $-4,55$ \\
\hline 72 & V. Fértil & 1 & $1-5$ & $-4,75$ & $-3,02$ & $-2,25$ & $-14,24$ & $-9,05$ & $-6,74$ \\
\hline 73 & P. Colgada & 2 & $2-1$ & $-4,80$ & $-3,79$ & $-1,69$ & $-14,40$ & $-11,38$ & $-5,08$ \\
\hline 74 & P. Colgada & 5 & $5-5$ & $-4,59$ & $-3,47$ & $-2,62$ & $-13,76$ & $-10,42$ & $-7,85$ \\
\hline 75 & V. Fértil & 3 & $3-2$ & $-4,17$ & $-4,24$ & $-2,60$ & $-12,52$ & $-12,71$ & $-7,80$ \\
\hline 76 & V. Fértil & 3 & $3-4$ & $-4,75$ & $-4,40$ & $-2,10$ & $-14,24$ & $-13,19$ & $-6,30$ \\
\hline
\end{tabular}

\section{DISCUSIÓN Y CONCLUSIONES}

La evaluación efectuada permite discriminar de entre los 76 árboles evaluados (candidatos y árboles de comparación) a los que presentan mejores expectativas para considerarlos como progenitores en un programa de mejoramiento genético, o como fuente directa de germoplasma para producuir plantas con algún nivel de mejoramiento en variables de interés para la utilización de chañar.

Debe sin embargo considerarse que los criterios de selección y evaluación utilizados se basan en supuestos de homogeneidad de sitio y coetaneidad entre los árboles, de modo que las diferencias entre ellos puedan artribuirse a componentes genéticos. El supuesto de homogeneidad ambiental puede considerarse cumplido, por cuanto en el método de selección empleado (árboles de comparación) las comparaciones se efectúan entre candidatos y vecinos que ocupan un mismo micrositio, es decir que se encuentran próximos entre sí, compartiendo la misma condición ambiental. No obstante, el requisito de coetaneidad puede ser menos riguroso, lo que determinaría que algunas diferencias de tamaño y producción de frutos obedezcan más a la diferencia de edad entre los árboles que a la regulación genética. 
Como quiera que sea, esta incertidumbre es comun en los inicios de los programas de mejoramiento, al efectuar selecciones masales en bosques naturales. En lo sucesivo, en la medida que se disponga de ensayos de progenies de los árboles ahora escogidos, podrán hacerse selecciones de segunda generación sobre sus hijos, las que resultarán más confiables. Estos mismos ensayos serán necesarios para estimar valores reales de heredabilidad, la cual requiere ensayos con estructura familiar conocida y diseños adecuados para poder estimarla.

Esas tres condiciones del ensayo de progenies; coetaneidad, homogeneidad ambiental y estructura familiar conocida, permitirán selecciones más robustas y con criterio genético en base a valores de mejora (breeding value) estimados por técnicas de genética cuantitativa.

El ensayo servirá también para estimar la heradabilidad y con ella calcular la ganancia genética esperada para las selecciones de seguda generación; esto representa un gran avance respecto al análisis actual el cual estima ganacias esperadas asumiendo valores probables de heredabilidad, pero sin conocerla. Por último el ensayo también permitirá calcular la ganancia genética real o lograda de los árboles ahora escogidos, parámetro mas confiable que la ganancia esperada que se puede estimar en esta fase inicial del programa.

\section{REFERENCIAS}

Gutiérrez, B., 2000. Ganancias Genéticas Esperadas para Poblaciones de Producción de Primera Generación de Nothofagus. En: Ipinza, R.; Gutiérrez, B. y Emhart, V. (eds.) Domesticación y Mejora Genética de Raulí y Roble. Universidad Austral de Chile-Instituto Forestal. Pp: 391-402.

Gutiérrez, B.; Gacitúa, S.; Perret, S.; Sandoval, A. y Curimil, M., 2013. Propagación de Especies Forestales Nativas de las Zonas Aridas y Semiáridas de Chile. Instituto Forestal. Manual № 47. Santiago, Chile. 142 p.

Ipinza, R.; Bello, A.; y Navarrete, M., 2000. Selección de Árboles Plus de Nothofagus alpina y N. obliqua. En: Ipinza, R.; Gutiérrez, B. y Emhart, V. (Eds.) Domesticación y Mejora Genética de Raulí y Roble. Universidad Austral de Chile-Instituto Forestal. Pp: 237-256.

Vallejos, J; Badilla, Y., Picado, F. y Murillo, O., 2010. Metodología para la Selección e Incorporación de Árboles Plus en Programas de Mejoramiento Genético Forestal. Agronomía Costarricense 34(1): 105-119. 